\title{
Implicit One-Step Block Hybrid Third-Derivative Method for the Direct Solution of Initial Value Problems of Second-Order Ordinary Differential Equations
}

\author{
Mohammad Alkasassbeh and Zurni Omar \\ Department of Mathematics, School of Quantitative Sciences, College of Arts and Sciences, Universiti Utara Malaysia, Sintok, Malaysia \\ Correspondence should be addressed to Mohammad Alkasassbeh; mfkasassbeh@yahoo.com
}

Received 25 August 2016; Revised 20 October 2016; Accepted 23 October 2016; Published 18 January 2017

Academic Editor: Mehmet Sezer

Copyright (C) 2017 Mohammad Alkasassbeh and Zurni Omar. This is an open access article distributed under the Creative Commons Attribution License, which permits unrestricted use, distribution, and reproduction in any medium, provided the original work is properly cited.

\begin{abstract}
A new one-step block method with generalized three hybrid points for solving initial value problems of second-order ordinary differential equations directly is proposed. In deriving this method, a power series approximate function is interpolated at $\left\{x_{n}, x_{n+r}\right\}$ while its second and third derivatives are collocated at all points $\left\{x_{n}, x_{n+r}, x_{n+s}, x_{n+t}, x_{n+1}\right\}$ in the given interval. The proposed method is then tested on initial value problems of second-order ordinary differential equations solved by other methods previously. The numerical results confirm the superiority of the new method to the existing methods in terms of accuracy.
\end{abstract}

\section{Introduction}

Numerous problems such as chemical kinetics, orbital dynamics, circuit and control theory, and Newton's second law applications involve second-order ordinary differential equations (ODEs). Normally, those equations have no analytical solutions. To approximate the solution of such problems several numerical methods were developed on the hands of many scholars such as [1-3].

Block methods for solving ODEs were first proposed by Milne ([4]). Later [5] adopted Milne's methods to provide starting values for predictor-corrector scheme. However, the block methods have some drawbacks and this led to the introduction of hybrid methods. According to [6], hybrid methods were initially introduced to overcome zero-stability barrier that occurred in block methods in Dahlquists ([7]). Besides the ability to change step size, the other benefit of these methods is utilizing data off-step points which contribute to the accuracy of the methods.

To increase the accuracy of the numerical methods further, researchers such as $[8,9]$ proposed high method derivative to overcome stiffness in ODEs. The former presented another type of hybrid methods called secondderivative methods, while the later proposed a Simpson's type second-derivative method for the solution of a stiff system of first-order IVPs. These scholars motivated us to develop a new generalized three-hybrid one-step third-derivative implicit method for solving second-order ODEs directly using the approach of interpolation and collocation for the general use to improve the efficiency of the approximate solution.

This article is organized as follows: in the coming section we demonstrate the derivation of the method, where we consider three off-step points through the approach of interpolation and collocation. The details of the analysis of the method are discussed in Section 3 which include zero stability, order, consistency, and convergence. In Section 4 some numerical problems are solved and the performance of the developed method is compared with other methods mentioned in literature. Finally, the conclusion is discussed in Section 5 .

\section{Development of the Method}

An approximate power series basis function taking the form

$$
p(x)=\sum_{j=0}^{2 v+u-1} a_{j}\left(\frac{x-x_{n}}{h}\right)^{j},
$$


where $u=2$ and $v=5$ are the number of interpolation and collocation points, respectively, is considered to be a solution to the following ODE:

$$
\begin{aligned}
y^{\prime \prime} & =f\left(x, y, y^{\prime}\right), \\
y(a) & =p_{0}, \\
y_{0}^{\prime}(a) & =p_{0}^{\prime},
\end{aligned}
$$

$$
x \in[a, b] .
$$

On derivation of (1) twice and thrice we obtain

$$
\begin{aligned}
p^{\prime \prime}(x) & =\sum_{j=2}^{2 v+u-1} \frac{a_{j} j !}{h^{2}(j-2) !}\left(\frac{x-x_{n}}{h}\right)^{j-2} \\
& =f\left(x, y, y^{\prime}\right), \\
p^{\prime \prime \prime}(x) & =\sum_{j=3}^{2 v+u-1} \frac{a_{j} j !}{h^{3}(j-3) !}\left(\frac{x-x_{n}}{h}\right)^{j-3} \\
& =g\left(x, y, y^{\prime}\right) .
\end{aligned}
$$

Interpolating (1) at $x_{n+\widehat{u}}=x_{n}+\widehat{u} h, \widehat{u}=\{0, r\}$ and collocating (3) at all points $x_{n+\widehat{v}}=x_{n}+\widehat{v} h, \widehat{v}=\{0, r, s, t, 1\}$, where $\{r, s, t\} \in$ $(0,1)$, a system of equations in matrix form is produced as below:

$$
A X=U,
$$

where

$$
A=\left[\begin{array}{l}
a_{0} \\
a_{1} \\
a_{2} \\
a_{3} \\
a_{4} \\
a_{5} \\
a_{6} \\
a_{7} \\
a_{8} \\
a_{9} \\
a_{10} \\
a_{11}
\end{array}\right],
$$

$X$

$$
=\left[\begin{array}{ccccccc}
1 & 0 & 0 & 0 & 0 & \cdots & 0 \\
1 & r & r^{2} & r^{3} & r^{4} & \cdots & r^{(2 v+u-1)} \\
0 & 0 & \frac{2 !}{0 ! h^{2}} & 0 & 0 & \cdots & 0 \\
0 & 0 & \frac{2 !}{0 ! h^{2}} & \frac{3 ! r}{1 ! h^{2}} & \frac{4 ! r^{2}}{2 ! h^{2}} & \cdots & \frac{(2 v+u-1) ! r^{(2 v+u-3)}}{(2 v+u-3) ! h^{2}} \\
0 & 0 & \frac{2 !}{0 ! h^{2}} & \frac{3 ! s}{1 ! h^{2}} & \frac{4 ! s^{2}}{2 ! h^{2}} & \cdots & \frac{(2 v+u-1) ! s^{(2 v+u-3)}}{(2 v+u-3) ! h^{2}} \\
0 & 0 & \frac{2 !}{0 ! h^{2}} & \frac{3 ! t}{1 ! h^{2}} & \frac{4 ! t^{2}}{2 ! h^{2}} & \cdots & \frac{(2 v+u-1) ! t^{(2 v+u-3)}}{(2 v+u-3) ! h^{2}} \\
0 & 0 & \frac{2 !}{0 ! h^{2}} & \frac{3 !}{1 ! h^{2}} & \frac{4 !}{2 ! h^{2}} & \cdots & \frac{(2 v+u-1) !}{(2 v+u-3) ! h^{2}} \\
0 & 0 & 0 & \frac{3 !}{0 ! h^{3}} & 0 & \cdots & 0 \\
0 & 0 & 0 & \frac{3 ! r}{0 ! h^{3}} & \frac{4 ! r^{2}}{1 ! h^{3}} & \cdots & \frac{(2 v+u-1) ! r^{(2 v+u-4)}}{(2 v+u-4) ! h^{3}} \\
0 & 0 & 0 & \frac{3 ! s}{0 ! h^{3}} & \frac{4 ! s^{2}}{1 ! h^{3}} & \cdots & \frac{(2 v+u-1) ! s^{(2 v+u-4)}}{(2 v+u-4) ! h^{3}} \\
0 & 0 & 0 & \frac{3 ! t}{0 ! h^{3}} & \frac{4 ! t^{2}}{1 ! h^{3}} & \cdots & \frac{(2 v+u-1) ! t^{(2 v+u-4)}}{(2 v+u-4) ! h^{3}} \\
0 & 0 & 0 & \frac{3 !}{0 ! h^{3}} & \frac{4 !}{1 ! h^{3}} & \cdots & \frac{(2 v+u-1) !}{(2 v+u-4) ! h^{3}}
\end{array}\right],
$$$$
U=\left[\begin{array}{c}
y_{n} \\
y_{n+r} \\
f_{n} \\
f_{n+r} \\
f_{n+s} \\
f_{n+t} \\
f_{n+1} \\
g_{n} \\
g_{n+r} \\
g_{n+s} \\
g_{n+t} \\
g_{n+1}
\end{array}\right] .
$$

Using matrix manipulation to solve (4) for the unknown coefficients $a_{j}^{\prime}$ s and then substituting them back into (1) yield

$$
\begin{aligned}
p(x)= & \sum_{i=0, r} \alpha_{i} y_{n+i}+h^{2}\left[\sum_{i=r, s, t} \beta_{i} f_{n+i}+\sum_{i=0}^{1} \beta_{i} f_{n+i}\right] \\
& +h^{3}\left[\sum_{i=r, s, t} \gamma_{i} g_{n+i}+\sum_{i=0}^{1} \gamma_{i} g_{n+i}\right],
\end{aligned}
$$

where $n=0,1,2, \ldots, N-1, h=x_{n}-x_{n-1}$ is the constant step size for the partition $\pi_{N}$ of the interval $[a, b]$ which 
is given by $\pi_{N}=\left[a=x_{0}<x_{1}<\cdots<x_{N-1}<\right.$ $\left.x_{N}=b\right], \alpha_{i}, \beta_{i}$, and $\gamma_{i}$ are undetermined constants listed in Appendix I in Supplementary Material available online at https://doi.org/10.1155/2017/8510948, $f_{n+i}=f(x+i h)$, and $g_{n+i}=d f\left(x_{n+i}, y_{n+i}, y_{n+i}^{\prime}\right) / d x$ whose first partial derivative is

$$
\begin{aligned}
p^{\prime}(x)= & \frac{1}{h} \sum_{i=0, r} \alpha_{i}^{\prime} y_{n+i}+h\left[\sum_{i=r, s, t} \beta_{i}^{\prime} f_{n+i}+\sum_{i=0}^{1} \beta_{i}^{\prime} f_{n+i}\right] \\
& +h^{2}\left[\sum_{i=r, s, t} \gamma_{i}^{\prime} g_{n+i}+\sum_{i=0}^{1} \gamma_{i}^{\prime} g_{n+i}\right] .
\end{aligned}
$$

Evaluating (6) at the noninterpolating points $\left\{x_{n+s}, x_{n+t}\right.$, $\left.x_{n+1}\right\}$ and (7) at all points $x_{n+i}, i=\{0, r, s, t, 1\}$, produces the following general equations in block form:

$$
A^{[0]} Y_{m}^{[1]}=A^{[1]} Y_{m}^{[0]}+\sum_{i=0}^{1} B^{[i]} F_{m}^{[i]}+\sum_{i=0}^{1} D^{[i]} G_{m}^{[i]},
$$

where $A^{[0]}$ is an $8 \times 8$ identity matrix and

$$
\begin{gathered}
A^{[1]}=\left[\begin{array}{llllllll}
0 & 0 & 0 & 1 & 0 & 0 & 0 & r h \\
0 & 0 & 0 & 1 & 0 & 0 & 0 & s h \\
0 & 0 & 0 & 1 & 0 & 0 & 0 & t h \\
0 & 0 & 0 & 1 & 0 & 0 & 0 & h \\
0 & 0 & 0 & 0 & 0 & 0 & 0 & 1 \\
0 & 0 & 0 & 0 & 0 & 0 & 0 & 1 \\
0 & 0 & 0 & 0 & 0 & 0 & 0 & 1 \\
0 & 0 & 0 & 0 & 0 & 0 & 0 & 1
\end{array}\right], \\
B^{[0]}
\end{gathered}=\left[\begin{array}{lllllllc}
0 & 0 & 0 & 0 & 0 & 0 & 0 & B_{18}^{[0]} \\
0 & 0 & 0 & 0 & 0 & 0 & 0 & B_{28}^{[0]} \\
0 & 0 & 0 & 0 & 0 & 0 & 0 & B_{38}^{[0]} \\
0 & 0 & 0 & 0 & 0 & 0 & 0 & B_{48}^{[0]} \\
0 & 0 & 0 & 0 & 0 & 0 & 0 & B_{58}^{[0]} \\
0 & 0 & 0 & 0 & 0 & 0 & 0 & B_{68}^{[0]} \\
0 & 0 & 0 & 0 & 0 & 0 & 0 & B_{78}^{[0]} \\
0 & 0 & 0 & 0 & 0 & 0 & 0 & B_{88}^{[0]}
\end{array}\right],
$$

$$
B^{[1]}=\left[\begin{array}{cccc}
B_{11}^{[1]} & B_{12}^{[1]} & B_{13}^{[1]} & B_{14}^{[1]} \\
B_{21}^{[1]} & B_{22}^{[1]} & B_{23}^{[1]} & B_{24}^{[1]} \\
B_{31}^{[1]} & B_{32}^{[1]} & B_{33}^{[1]} & B_{34}^{[1]} \\
B_{41}^{[1]} & B_{42}^{[1]} & B_{43}^{[1]} & B_{44}^{[1]} \\
B_{51}^{[1]} & B_{52}^{[1]} & B_{53}^{[1]} & B_{54}^{[1]} \\
B_{61}^{[1]} & B_{62}^{[1]} & B_{63}^{[1]} & B_{64}^{[1]} \\
B_{71}^{[1]} & B_{72}^{[1]} & B_{73}^{[1]} & B_{74}^{[1]} \\
B_{81}^{[1]} & B_{82}^{[1]} & B_{83}^{[1]} & B_{84}^{[1]}
\end{array}\right],
$$$$
D^{[0]}=\left[\begin{array}{lllllllc}
0 & 0 & 0 & 0 & 0 & 0 & 0 & D_{18}^{[0]} \\
0 & 0 & 0 & 0 & 0 & 0 & 0 & D_{28}^{[0]} \\
0 & 0 & 0 & 0 & 0 & 0 & 0 & D_{38}^{[0]} \\
0 & 0 & 0 & 0 & 0 & 0 & 0 & D_{48}^{[0]} \\
0 & 0 & 0 & 0 & 0 & 0 & 0 & D_{58}^{[0]} \\
0 & 0 & 0 & 0 & 0 & 0 & 0 & D_{68}^{[0]} \\
0 & 0 & 0 & 0 & 0 & 0 & 0 & D_{78}^{[0]} \\
0 & 0 & 0 & 0 & 0 & 0 & 0 & D_{88}^{[0]}
\end{array}\right],
$$$$
D^{[1]}=\left[\begin{array}{llll}
D_{11}^{[1]} & D_{12}^{[1]} & D_{13}^{[1]} & D_{14}^{[1]} \\
D_{21}^{[1]} & D_{22}^{[1]} & D_{23}^{[1]} & D_{24}^{[1]} \\
D_{31}^{[1]} & D_{32}^{[1]} & D_{33}^{[1]} & D_{34}^{[1]} \\
D_{41}^{[1]} & D_{42}^{[1]} & D_{43}^{[1]} & D_{44}^{[1]} \\
D_{51}^{[1]} & D_{52}^{[1]} & D_{53}^{[1]} & D_{54}^{[1]} \\
D_{61}^{[1]} & D_{62}^{[1]} & D_{63}^{[1]} & D_{64}^{[1]} \\
D_{71}^{[1]} & D_{72}^{[1]} & D_{73}^{[1]} & D_{74}^{[1]} \\
D_{81}^{[1]} & D_{82}^{[1]} & D_{83}^{[1]} & D_{84}^{[1]}
\end{array}\right] .
$$

Their entries are listed in Appendix II in Supplementary Material, while the vectors $Y_{m}^{[0]}, Y_{m}^{[1]}, F_{m}^{[0]}, F_{m}^{[1]}, G_{m}^{[0]}, G_{m}^{[1]}$ are defined as follows:

$$
Y_{m}^{[0]}=\left[\begin{array}{c}
y_{n-t} \\
y_{n-s} \\
y_{n-r} \\
y_{n} \\
y_{n-t}^{\prime} \\
y_{n-s}^{\prime} \\
y_{n-r}^{\prime} \\
y_{n}^{\prime}
\end{array}\right],
$$




$$
\begin{array}{r}
Y_{m}^{[1]}=\left[\begin{array}{c}
y_{n+r} \\
y_{n+s} \\
y_{n+t} \\
y_{n+1} \\
y_{n+r}^{\prime} \\
y_{n+s}^{\prime} \\
y_{n+t}^{\prime} \\
y_{n+1}^{\prime}
\end{array}\right], \quad \begin{array}{r}
\text { To illustrate that the root of the first characteristic } \\
\text { equation satisfies the prior definition we assume that }\{r, s, t\} \in \\
(0,1) \text { and hence }
\end{array} \\
\rho(R)=\operatorname{det}\left[R A^{[0]}-A^{[1]}\right] \\
{\left[\begin{array}{c}
f_{n-7} \\
f_{n-6} \\
f_{n-5} \\
f_{n+4}
\end{array}\right]} \\
\quad=\left|\begin{array}{rrrrrrrr}
R & 0 & 0 & -1 & 0 & 0 & 0 & -r h \\
0 & R & 0 & -1 & 0 & 0 & 0 & -s h \\
0 & 0 & R & -1 & 0 & 0 & 0 & -t h \\
0 & 0 & 0 & R-1 & 0 & 0 & 0 & -h \\
0 & 0 & 0 & 0 & R & 0 & 0 & -1 \\
0 & 0 & 0 & 0 & 0 & R & 0 & -1 \\
0 & 0 & 0 & 0 & 0 & 0 & R & -1 \\
0 & 0 & 0 & 0 & 0 & 0 & 0 & R-1
\end{array}\right|=0
\end{array}
$$$$
F_{m}^{[0]}=\left[\begin{array}{c}
f_{n-7} \\
f_{n-6} \\
f_{n-5} \\
f_{n-4} \\
f_{n-3} \\
f_{n-2} \\
f_{n-1} \\
f_{n}
\end{array}\right] \text {, }
$$$$
F_{m}^{[1]}=\left[\begin{array}{c}
f_{n+r} \\
f_{n+s} \\
f_{n+t} \\
f_{n+1}
\end{array}\right] \text {, }
$$$$
G_{m}^{[0]}=\left[\begin{array}{c}
g_{n-7} \\
g_{n-6} \\
g_{n-5} \\
g_{n-4} \\
g_{n-3} \\
g_{n-2} \\
g_{n-1} \\
g_{n}
\end{array}\right],
$$$$
G_{m}^{[1]}=\left[\begin{array}{c}
g_{n+r} \\
g_{n+s} \\
g_{n+t} \\
g_{n+1}
\end{array}\right] .
$$

\section{Analysis of the Method}

\subsection{Zero Stability}

Definition 1. The hybrid block method formula (8) is said to be zero stable if no root of the first characteristic equation $\rho(R)$ has modulus greater than one; that is, $\left|R_{m}\right| \leqslant 1$ and if $R_{m}=1$ then the multiplicity of $R_{m}$ must not exceed two. which imply that

$$
R^{6}(R-1)^{2}=0 \Longrightarrow R_{i}= \begin{cases}0, & \text { if } i=1(1) 6 \\ 1, & \text { if } i=7,8\end{cases}
$$

As a result, the developed method is zero stable.

3.2. Order of the Method. The linear operator $\Delta$ associated with the hybrid block methods formula (8) is defined as

$$
\begin{aligned}
\Delta\{y(x) ; h\}= & A^{[0]} Y_{m}^{[1]}-A^{[1]} Y_{m}^{[0]}-\sum_{i=0}^{1} B^{[i]} F_{m}^{[i]} \\
& -\sum_{i=0}^{1} D^{[i]} G_{m}^{[i]} .
\end{aligned}
$$

Expanding the above equation in Taylor series and combining like terms we wind up with

$$
\begin{aligned}
\Delta\{y(x) ; h\}= & \widehat{C}_{0} h^{0} y(x)+\widehat{C}_{1} h^{1} y^{\prime}(x)+\widehat{C}_{2} h^{2} y^{\prime \prime}(x) \\
& +\cdots+\widehat{C}_{p+2} h^{p+2} y^{(p+2)}(x)+\cdots
\end{aligned}
$$

According to $[6,10]$ method $(8)$ is said to be of order $p$ if

$$
\begin{aligned}
\widehat{C}_{0} & =\widehat{C}_{1}=\cdots=\widehat{C}_{p+1}=0, \\
\widehat{C}_{p+2} & \neq 0 .
\end{aligned}
$$

The term $\widehat{C}_{p+2}$ is called the error constant and the local truncation error is given by

$$
t_{n+k}=\widehat{C}_{p+2} y^{(p+2)} h^{p+2}+O\left(h^{p+3}\right) .
$$

Comparing like terms of $y^{(i)}$ and $h^{i}$ in (14) produces the coefficients $\widehat{C}_{0}=\widehat{C}_{1}=\cdots=\widehat{C}_{11}=0$ with vector of error constants

$$
\widehat{C}_{12}=\left[\begin{array}{llllllll}
\widehat{C}_{12}^{[1]} & \widehat{C}_{12}^{[2]} & \widehat{C}_{12}^{[3]} & \widehat{C}_{12}^{[4]} & \widehat{C}_{12}^{[5]} & \widehat{C}_{12}^{[6]} & \widehat{C}_{12}^{[7]} & \widehat{C}_{12}^{[8]}
\end{array}\right]^{T},
$$


where

$$
\begin{aligned}
& \widehat{C}_{12}^{[1]}=\frac{h^{12} r^{6} y^{12}}{100590336000}\left(\left(14 r^{6}-42 r^{5} s-42 r^{5} t-42 r^{5}\right.\right. \\
& +33 r^{4} s^{2}+132 r^{4} s t+132 r^{4} s+33 r^{4} t^{2}+132 r^{4} t \\
& +33 r^{4}-110 r^{3} s^{2} t-110 r^{3} s^{2}-110 r^{3} s t^{2}-440 r^{3} s t \\
& -110 r^{3} s-110 r^{3} t^{2}-110 r^{3} t+99 r^{2} s^{2} t^{2}+396 r^{2} s^{2} t \\
& +99 r^{2} s^{2}+396 r^{2} s t^{2}+396 r^{2} s t+99 r^{2} t^{2}-396 r s^{2} t^{2} \\
& \left.\left.-396 r s^{2} t-396 r s t^{2}+462 s^{2} t^{2}\right)\right) \text {, } \\
& \widehat{C}_{12}^{[2]}=\frac{h^{12} s^{6} y^{12}}{100590336000}\left(\left(33 r^{2} s^{4}-110 r^{2} s^{3} t-110 r^{2} s^{3}\right.\right. \\
& +99 r^{2} s^{2} t^{2}+396 r^{2} s^{2} t+99 r^{2} s^{2}-396 r^{2} s t^{2} \\
& -396 r^{2} s t+462 r^{2} t^{2}-42 r s^{5}+132 r s^{4} t+132 r s^{4} \\
& -110 r s^{3} t^{2}-440 r s^{3} t-110 r s^{3}+396 r s^{2} t^{2} \\
& +396 r s^{2} t-396 r s t^{2}+14 s^{6}-42 s^{5} t-42 s^{5} \\
& +33 s^{4} t^{2}+132 s^{4} t+33 s^{4}-110 s^{3} t^{2}-110 s^{3} t \\
& \left.\left.+99 s^{2} t^{2}\right)\right) \text {, } \\
& \widehat{C}_{12}^{[3]}=\frac{h^{12} t^{6} y^{12}}{100590336000}\left(\left(99 r^{2} s^{2} t^{2}-396 r^{2} s^{2} t\right.\right. \\
& +462 r^{2} s^{2}-110 r^{2} s t^{3}+396 r^{2} s t^{2}-396 r^{2} s t \\
& +33 r^{2} t^{4}-110 r^{2} t^{3}+99 r^{2} t^{2}-110 r s^{2} t^{3}+396 r s^{2} t^{2} \\
& -396 r s^{2} t+132 r s t^{4}-440 r s t^{3}+396 r s t^{2}-42 r t^{5} \\
& +132 r t^{4}-110 r t^{3}+33 s^{2} t^{4}-110 s^{2} t^{3}+99 s^{2} t^{2} \\
& \left.\left.-42 s t^{5}+132 s t^{4}-110 s t^{3}+14 t^{6}-42 t^{5}+33 t^{4}\right)\right) \text {, } \\
& \widehat{C}_{12}^{[4]}=\frac{h^{12} y^{12}}{100590336000}\left(\left(462 r^{2} s^{2} t^{2}-396 r^{2} s^{2} t\right.\right. \\
& +99 r^{2} s^{2}-396 r^{2} s t^{2}+396 r^{2} s t-110 r^{2} s+99 r^{2} t^{2} \\
& -110 r^{2} t+33 r^{2}-396 r s^{2} t^{2}+396 r s^{2} t-110 r s^{2} \\
& +396 r s t^{2}-440 r s t+132 r s-110 r t^{2}+132 r t \\
& -42 r+99 s^{2} t^{2}-110 s^{2} t+33 s^{2}-110 s t^{2}+132 s t \\
& \left.\left.-42 s+33 t^{2}-42 t+14\right)\right) \text {, } \\
& \widehat{C}_{12}^{[5]}=\frac{h^{11} r^{5} y^{12}}{50295168000}\left(\left(28 r^{6}-77 r^{5} s-77 r^{5} t-77 r^{5}\right.\right. \\
& +55 r^{4} s^{2}+220 r^{4} s t+220 r^{4} s+55 r^{4} t^{2}+220 r^{4} t \\
& +55 r^{4}-165 r^{3} s^{2} t-165 r^{3} s^{2}-165 r^{3} s t^{2}-660 r^{3} s t
\end{aligned}
$$

$$
\begin{aligned}
& -165 r^{3} s-165 r^{3} t^{2}-165 r^{3} t+132 r^{2} s^{2} t^{2} \\
& +528 r^{2} s^{2} t+132 r^{2} s^{2}+528 r^{2} s t^{2}+528 r^{2} s t \\
& +132 r^{2} t^{2}-462 r s^{2} t^{2}-462 r s^{2} t-462 r s t^{2} \\
& \left.\left.+462 s^{2} t^{2}\right)\right) \text {, } \\
& \widehat{C}_{12}^{[6]}=\frac{h^{11} s^{5} y^{12}}{50295168000}\left(\left(55 r^{2} s^{4}-165 r^{2} s^{3} t-165 r^{2} s^{3}\right.\right. \\
& +132 r^{2} s^{2} t^{2}+528 r^{2} s^{2} t+132 r^{2} s^{2}-462 r^{2} s t^{2} \\
& -462 r^{2} s t+462 r^{2} t^{2}-77 r s^{5}+220 r s^{4} t+220 r s^{4} \\
& -165 r s^{3} t^{2}-660 r s^{3} t-165 r s^{3}+528 r s^{2} t^{2} \\
& +528 r s^{2} t-462 r s t^{2}+28 s^{6}-77 s^{5} t-77 s^{5} \\
& +55 s^{4} t^{2}+220 s^{4} t+55 s^{4}-165 s^{3} t^{2}-165 s^{3} t \\
& \left.\left.+132 s^{2} t^{2}\right)\right) \text {, } \\
& \widehat{C}_{12}^{[7]}=\frac{h^{11} t^{5} y^{12}}{50295168000}\left(\left(132 r^{2} s^{2} t^{2}-462 r^{2} s^{2} t\right.\right. \\
& +462 r^{2} s^{2}-165 r^{2} s t^{3}+528 r^{2} s t^{2}-462 r^{2} s t \\
& +55 r^{2} t^{4}-165 r^{2} t^{3}+132 r^{2} t^{2}-165 r s^{2} t^{3} \\
& +528 r s^{2} t^{2}-462 r s^{2} t+220 r s t^{4}-660 r s t^{3} \\
& +528 r s t^{2}-77 r t^{5}+220 r t^{4}-165 r t^{3}+55 s^{2} t^{4} \\
& -165 s^{2} t^{3}+132 s^{2} t^{2}-77 s t^{5}+220 s t^{4}-165 s t^{3} \\
& \left.\left.+28 t^{6}-77 t^{5}+55 t^{4}\right)\right) \\
& \widehat{C}_{12}^{[8]}=\frac{h^{11} y^{12}}{50295168000}\left(\left(462 r^{2} s^{2} t^{2}-462 r^{2} s^{2} t\right.\right. \\
& +132 r^{2} s^{2}-462 r^{2} s t^{2}+528 r^{2} s t-165 r^{2} s \\
& +132 r^{2} t^{2}-165 r^{2} t+55 r^{2}-462 r s^{2} t^{2}+528 r s^{2} t \\
& -165 r s^{2}+528 r s t^{2}-660 r s t+220 r s-165 r t^{2} \\
& +220 r t-77 r+132 s^{2} t^{2}-165 s^{2} t+55 s^{2}-165 s t^{2} \\
& \left.\left.+220 s t-77 s+55 t^{2}-77 t+28\right)\right),
\end{aligned}
$$

which conclude that the order of the developed method is $p=$ 10.

\subsection{Consistency}

Definition 2. A block method is said to be consistent if its order $p$ is greater than one. 
TABLE 1: Comparison of the proposed method with [11].

\begin{tabular}{lcccc}
\hline$X$ value & Exact solution & $\begin{array}{c}\text { Computed solution for } \\
r=1 / 2 ; s=2 / 3 ; t=3 / 4\end{array}$ & Error in new method & Error for [11] \\
\hline 0.100 & 0.904837418035959520 & 0.904837418035948970 & $1.054712 E(-14)$ & $2: 05 E(-11)$ \\
0.200 & 0.818730753077981820 & 0.818730753077964060 & $1.776357 E(-14)$ & $4: 39 E(-11)$ \\
0.300 & 0.740818220681717770 & 0.740818220681694340 & $2.342571 E(-14)$ & $6: 55 E(-11)$ \\
0.400 & 0.670320046035639330 & 0.670320046035611350 & $2.797762 E(-14)$ & $8: 38 E(-11)$ \\
0.500 & 0.606530659712633420 & 0.606530659712602120 & $3.130829 E(-14)$ & $9: 86 E(-11)$ \\
0.600 & 0.548811636094026500 & 0.548811636093992530 & $3.397282 E(-14)$ & $1: 10 E(-10)$ \\
0.700 & 0.496585303791409530 & 0.496585303791373890 & $3.563816 E(-14)$ & $1: 19 E(-10)$ \\
0.800 & 0.449328964117221620 & 0.449328964117184870 & $3.674838 E(-14)$ & $1: 28 E(-10)$ \\
0.900 & 0.406569659740599170 & 0.406569659740561860 & $3.741452 E(-14)$ \\
1.000 & 0.367879441171442330 & 0.367879441171404920 & $1: 30 E(-10)$ \\
\hline
\end{tabular}

TABLE 2: Comparison of the proposed method with [12].

\begin{tabular}{lcccc}
\hline$X$ value & Exact solution & $\begin{array}{c}\text { Computed solution for } \\
r=1 / 4 ; s=1 / 2 ; t=3 / 4\end{array}$ & Error in new method & Error for $[12]$ \\
\hline 0.100 & 1.050041729278491400 & 1.050041729278491200 & $2.220446 E(-16)$ & $1.1102 E(-15)$ \\
0.200 & 1.100335347731075600 & 1.100335347731075300 & $2.220446 E(-16)$ & $5.9952 E(-15)$ \\
0.300 & 1.151140435936466800 & 1.151140435936466100 & $6.661338 E(-16)$ & $7.1054 E(-14)$ \\
0.400 & 1.202732554054082100 & 1.202732554054081000 & $1.110223 E(-15)$ & $1.1568 E(-13)$ \\
0.500 & 1.255412811882995200 & 1.255412811882994800 & $4.440892 E(-16)$ & $1.1990 E(-13)$ \\
0.600 & 1.309519604203111900 & 1.309519604203112800 & $8.881784 E(-16)$ & $6.8567 E(-13)$ \\
0.700 & 1.365443754271396400 & 1.365443754271398000 & $4.4554312 E(-15)$ & $3.4754 E(-12)$ \\
0.800 & 1.423648930193601900 & 1.423648930193606400 & $8.659740 E(-15)$ & $1.2219 E(-11)$ \\
0.900 & 1.484700278594052000 & 1.484700278594060600 & $1.265654 E(-14)$ & $3.7282 E(-11)$ \\
1.000 & 1.549306144334055000 & 1.549306144334067700 & &
\end{tabular}

Consistency property is achieved for the hybrid block method from the above analysis since the order $p=10 \geq 1$.

\subsection{Convergence}

Theorem 3 (see [16]). Consistency and zero stability are sufficient conditions for a linear multistep method to be convergent

The hybrid block method equation (8) is convergent since it fulfills both the consistency and zero-stability conditions.

\section{Numerical Examples}

In this section, the efficiency and the performance of the general three-hybrid one-step implicit hybrid block method (8) with order $p=10$ is investigated on five test problems. The first example is highly stiff linear IVP problem with step size $h=1 / 10$, the second is nonlinear IVP with $h=5 / 100$, the third is linear with $h=1 / 100$, the fourth is a nonlinear system with $h=1 / 10$, and finally the fifth is a nonlinear undamped Duffing equation with $h=\pi / 5$. It is worth mentioning that this method works even for large interval and different values of step size. The values mentioned in this article are chosen just for the sake of comparison with the existing methods only.

Problem 4. $f\left(x, y, y^{\prime}\right)=-1001 y^{\prime}-1000 y, y(0)=1, y^{\prime}(0)=$ $-1,0 \leq x \leq 1$.

Exact Solution. $y=e^{-x}$ with $h=1 / 10$.

Source (see [11]). See Table 1.

Problem 5. $f\left(x, y, y^{\prime}\right)=x\left(y^{\prime}\right)^{2}, y(0)=1, y^{\prime}(0)=1 / 2,0 \leq$ $x \leq 1$.

Exact Solution. $y=1+\ln ((2+x) /(2-x))$ with $h=5 / 100$.

Source (see [12]). See Table 2.

Problem 6. $f\left(x, y, y^{\prime}\right)=y^{\prime}, y(0)=0, y^{\prime}(1)=-1,0 \leq x \leq 1$.

Exact Solution. $y=1-e^{x}$ with $h=1 / 100$.

Source (see [13]). See Table 3.

Problem 7 (a system of nonlinear IVP). $y_{1}^{\prime \prime}=1-\cos (x)+$ $\sin \left(y_{2}^{\prime}\right)+\cos \left(y_{2}^{\prime}\right), y_{1}(0)=0, y_{1}^{\prime}(0)=0,0 \leq x \leq 1$. 
TABLE 3: Comparison of the proposed method with [13].

\begin{tabular}{lcccc}
\hline$X$ value & Exact solution & $\begin{array}{c}\text { Computed solution for } \\
r=1 / 5 ; s=1 / 3 ; t=1 / 2\end{array}$ & Error in new method & Error for $[13]$ \\
\hline 0.100 & -0.105170918075647710 & -0.105170918075647660 & $5.551115 E(-17)$ & $2.2360 E(-13)$ \\
0.200 & -0.221402758160169850 & -0.221402758160169990 & $1.387779 E(-16)$ & $3.6425 E(-12)$ \\
0.300 & -0.349858807576003180 & -0.349858807576003520 & $3.330669 E(-16)$ & $8.6628 E(-12)$ \\
0.400 & -0.491824697641270570 & -0.491824697641271070 & $4.996004 E(-16)$ & $7.1338 E(-11)$ \\
0.500 & -0.648721270700128640 & -0.648721270700129420 & $1.332268 E(-15)$ & $2.0317 E(-11)$ \\
0.600 & -0.822118800390509550 & -0.822118800390510880 & $1.776357 E(-15)$ & $3.2476 E(-11)$ \\
0.700 & -1.013752707470477500 & -1.013752707470479300 & $2.886580 E(-15)$ & $4.5463 E(-11)$ \\
0.800 & -1.225540928492468800 & -1.225540928492471600 & $3.774758 E(-15)$ & $8.1781 E(-11)$ \\
0.900 & -1.459603111156951200 & -1.459603111156955000 & $5.107026 E(-15)$ & $8.2113 E(-11)$ \\
1.000 & -1.718281828459047300 & -1.718281828459052400 & &
\end{tabular}

TABLE 4: Exact and approximate solutions for solving $y_{1}$ using the developed method.

\begin{tabular}{lcccr}
\hline$X$ value & Exact solution & Approximate solution & Error in developed method & Error in $[14]$ \\
\hline 0.200 & 0.980066577841241630 & 0.980066580773730210 & $2.93 E(-09)$ & $3.331 E(-08)$ \\
0.400 & 0.921060994002885100 & 0.921060786088783830 & $2.08 E(-07)$ & $7.27 E(-07)$ \\
0.600 & 0.825335614909678330 & 0.825334546366523920 & $1.07 E(-06)$ & $3.09 E(-06)$ \\
0.800 & 0.696706709347165500 & 0.696703745729634890 & $2.96 E(-06)$ & $8.01 E(-06)$ \\
1.000 & 0.540302305868139770 & 0.540296131232123940 & $6.17 E(-06)$ & $1.60 E(-05)$ \\
\hline
\end{tabular}

TABLE 5: Exact and approximate solutions for solving $y_{2}$ using the developed method.

\begin{tabular}{lcccr}
\hline$X$ value & Exact solution & Approximate solution & Error in developed method & Error in $[14]$ \\
\hline 0.200 & 0.408407044966673130 & 0.628318530716756810 & $1.20 E(-12)$ & $5.74 E(-12)$ \\
0.400 & 1.036725575684631900 & 1.256637061492416500 & $5.65 E(-10)$ & $3.40 E(-10)$ \\
0.600 & 1.665044106402590500 & 1.884955593121642800 & $9.68 E(-10)$ & $3.48 E(-09)$ \\
0.800 & 2.293362637120548900 & 2.513274128242366100 & $5.37 E(-09)$ & $1.66 E(-08)$ \\
1.000 & 2.921681167838507500 & 3.141592671767204200 & $1.82 E(-08)$ & $5.24 E(-08)$ \\
\hline
\end{tabular}

TABLE 6: Comparison of the proposed method with [15].

\begin{tabular}{lcccr}
\hline$X$ value & Exact solution & Approximate solution & Error in developed method & Error in [15] \\
\hline$\pi$ & -0.200326851873144250 & -0.200326851873131260 & $1.30 E(-14)$ & $2.77 E(-6)$ \\
$2 \pi$ & 0.200027330586423440 & 0.200027330586373150 & $5.03 E(-14)$ & $2.97 E(-8)$ \\
$4 \pi$ & 0.198830853474466220 & 0.198830853474288970 & $1.77 E(-13)$ & $1.16 E(-7)$ \\
$6 \pi$ & 0.196842430954904000 & 0.196842430954587670 & $3.16 E(-13)$ & $2.53 E(-7)$ \\
$8 \pi$ & 0.194070581011836470 & 0.194070581011452750 & $3.84 E(-13)$ & $4.28 E(-7)$ \\
$10 \pi$ & 0.190527147620306290 & 0.190527147620002170 & $3.04 E(-13)$ & $6.29 E(-7)$ \\
\hline
\end{tabular}

$y_{2}^{\prime \prime}=1 /\left(4+y_{1}^{2}\right)-1 /\left(5-(\sin (x))^{2}\right), y_{2}(0)=0, y_{2}^{\prime}(0)=\pi$ $0 \leq x \leq 1$.

Exact Solutions. $y_{1}=\cos (x), y_{2}=\pi x$, with $h=(1 / 10)(r=$ $1 / 10, s=3 / 10, t=3 / 5)$; see Tables 4 and 5 .

Problem 8 (the nonlinear undamped Duffing equation). $y^{\prime \prime}+$ $y+y^{3}=Q \cos (\omega x), y(0)=k_{0}, y^{\prime}(0)=0,0 \leq x \leq 10 \pi$.

Exact Solutions. $y(x)=k_{1} \cos (\omega x)+k_{2} \cos (3 \omega x)+$ $k_{3} \cos (5 \omega x)+k_{4} \cos (7 \omega x)$, where $\omega=1.01, Q=0.002$, $k_{0}=0.200426728069, k_{1}=0.200179477536, k_{2}=$
$0.246946143 e(-3), k_{3}=0.304016 e(-6), k_{4}=0.374 e(-9)$, and $h=\pi / 5 .(r=1 / 4, s=1 / 2, t=3 / 4)$; see Table 6 .

\section{Conclusion}

A general three-hybrid one-step block method of order 10 has been proposed for the direct solution of general second-order ODEs. The developed method is tested on five different problems. Numerical analysis shows that the developed method is consistent and zero stable which conclude its convergence. The computed results are then compared with the results of existing methods in terms of error by considering different 
values of $r, s$, and $t$. The new method is found to have superiority over them as shown in Tables 1-6.

\section{Competing Interests}

The authors declare that there is no conflict of interests regarding the publication of this paper.

\section{Acknowledgments}

The authors thank Universiti Utara Malaysia for the financial support of the publication of this article.

\section{References}

[1] M. M. Chawla and S. R. Sharma, "Families of three-stage third order Runge-Kutta-Nystrom methods for $y^{\prime \prime}=f\left(x, y, y^{\prime}\right)$," The Journal of the Australian Mathematical Society. Series B. Applied Mathematics, vol. 26, no. 3, pp. 375-386, 1985.

[2] E. Hairer and G. Wanner, "A theory for Nyström methods," Numerische Mathematik, vol. 25, no. 4, pp. 383-400, 1976.

[3] J. Vigo-Aguiar and H. Ramos, "Variable stepsize implementation of multistep methods for $\mathrm{y}^{\prime \prime}=\mathrm{f}\left(\mathrm{x}, \mathrm{y}, \mathrm{y}^{\prime}\right)$," Journal of Computational and Applied Mathematics, vol. 192, no. 1, pp. 114131, 2006.

[4] W. E. Milne, Numerical Solution of Differential Equations, John Wiley \& Sons, New York, NY, USA, 1953.

[5] R. J. Barkley, "A Runge-kutta for all seasons," SIAM Review, vol. 9, no. 3, pp. 417-452, 1967.

[6] J. D. Lambert, Computational Methods in Ordinary Differential Equations, John Wiley \& Sons, New York, NY, USA, 1973.

[7] G. Dahlquist, "Convergence and stability in the numerical integration of ordinary differential equations," Mathematica Scandinavica, vol. 4, pp. 33-53, 1956.

[8] F. F. Ngwane and S. N. Jator, "Block hybrid-second derivative method for stiff systems," International Journal of Pure and Applied Mathematics, vol. 80, no. 4, pp. 543-559, 2012.

[9] R. K. Sahi, S. N. Jator, and N. A. Khan, "A Simpson's-type second derivative method for stiff systems," International Journal of Pure and Applied Mathematics, vol. 81, no. 4, pp. 619-633, 2012.

[10] S. O. Fatunla, Numerical Methods for Fatunla Initial Value Problems in Ordinary Differential Equations, Academic Press, New York, NY, USA, 1988.

[11] O. Adeniran and B. Ogundare, "An efficient hybrid numerical scheme for solving general second order initial value problems (IVPs)," International Journal of Applied Mathematical Research, vol. 4, no. 2, pp. 411-419, 2015.

[12] A. A. James, A. O. Adesanya, and S. Joshua, "Continuous block method for the solution of second order initial value problems of ordinary differential equation," International Journal of Pure and Applied Mathematics, vol. 83, no. 3, pp. 405-416, 2013.

[13] O. O. Olanegan, D. O. Awoyemi, B. G. Ogunware, and F. O. Obarhua, "Continuous double-hybrid point method for the solution of second order ordinary differential equations," International Journal of Advanced Scientific and Technical Research, vol. 5, no. 2, pp. 549-562, 2015.

[14] R. F. Abdelrahim and Z. Omar, "Direct solution of secondorder ordinary differential equation using a single-step hybrid block method of order five," Mathematical and Computational Applications, vol. 21, no. 2, pp. 1-12, 2016.
[15] S. N. Jator, "Block third derivative method based on trigonometric polynomials for periodic initial-value problems," Afrika Matematika, vol. 27, no. 3-4, pp. 365-377, 2016.

[16] P. Henrici, Discrete Variable Methods in ODEs, John Wiley \& Sons, New York, NY, USA, 1962. 


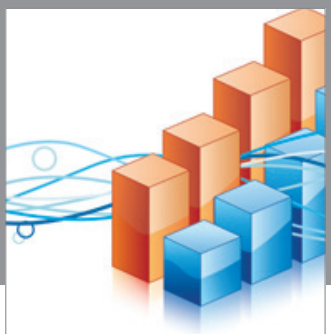

Advances in

Operations Research

vatem alat4

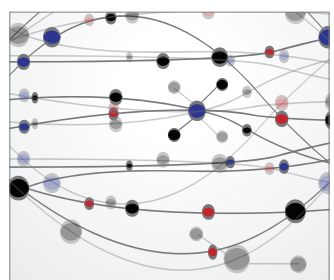

\section{The Scientific} World Journal
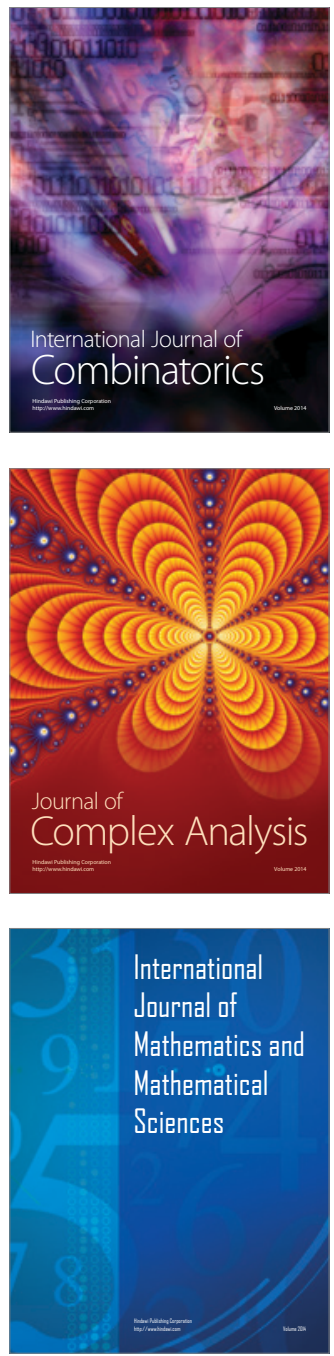
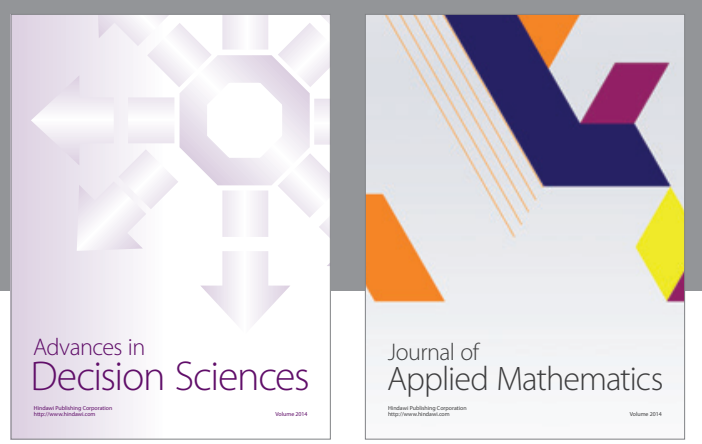

Algebra

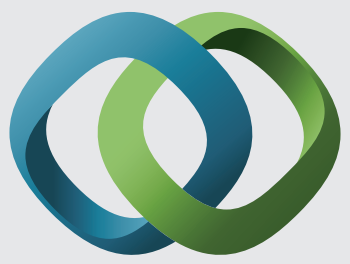

\section{Hindawi}

Submit your manuscripts at

https://www.hindawi.com
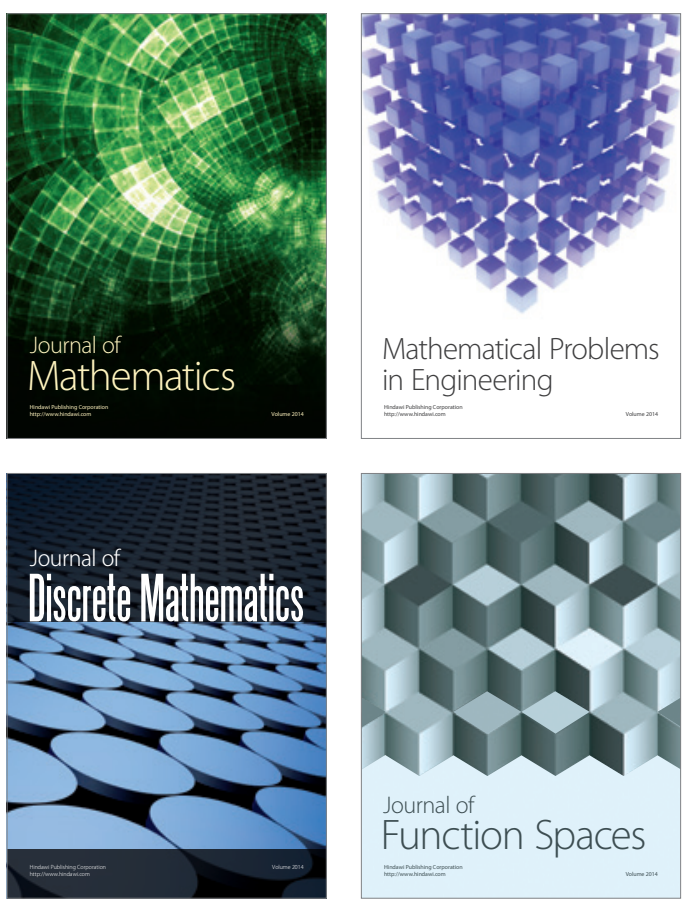

Mathematical Problems in Engineering
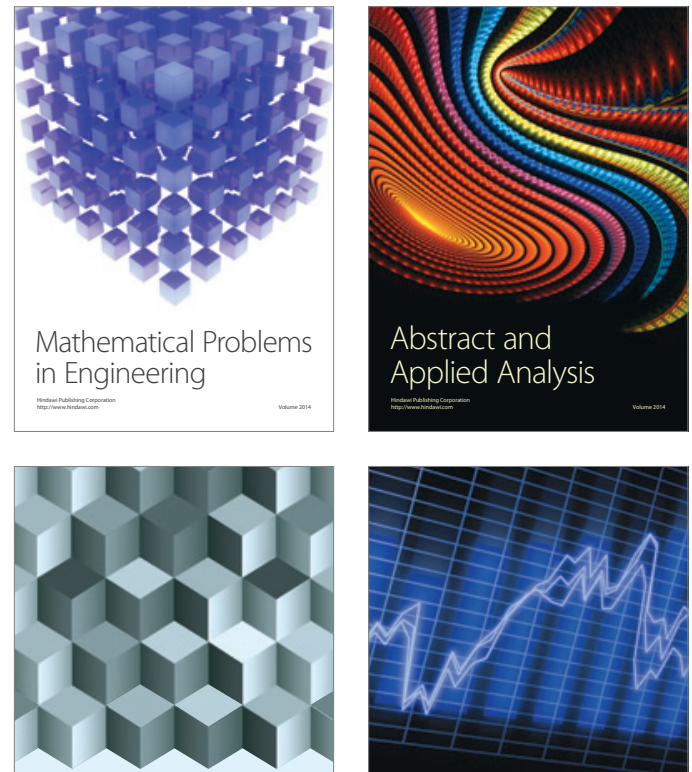

Journal of

Function Spaces

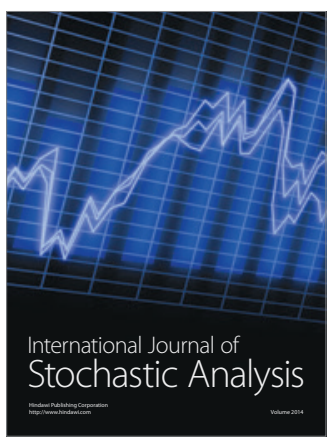

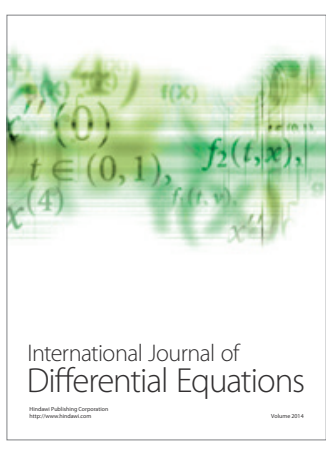
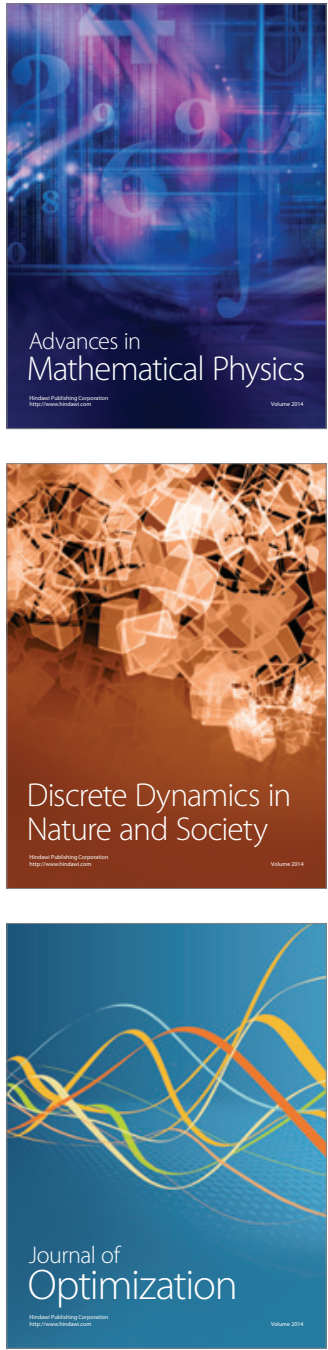